\title{
Model Predictive Control of Voltages in Active Distribution Networks
}

\author{
Gustavo Valverde, Member, IEEE, and Thierry Van Cutsem, Fellow, IEEE
}

\begin{abstract}
This paper presents a centralized control scheme to regulate distribution network voltages in the presence of high penetration of distributed generation. The approach is inspired of Model Predictive Control in order to compensate for modeling inaccuracies and measurement noise. The control actions, calculated from a multi-step optimization, are updated and corrected by real-time measurements. The proposed controller uses a linear model to predict the behavior of the system and the optimization is solved using quadratic programming. The proposed corrective control has been tested in a 11-kV distribution network including 75 nodes and hosting 22 distributed generating units.
\end{abstract}

Index Terms-Smart grids, voltage control, distributed generation, model predictive control, quadratic programming.

\section{INTRODUCTION}

$\mathbf{T}$ HE rapid proliferation of distributed energy sources has brought new operational problems in distribution networks. Temporary over/under voltages and congested lines are part of the negative effects caused by the intermittent production of Distributed Generation (DG).

Typical control actions such as Load Tap Changer (LTC) and capacitors switching are not flexible enough to mitigate the violations of voltages. In addition, reinforcing the network to deal with these temporary situations is not economically viable for the Distribution System Operator (DSO). Based on this, more flexible and coordinated control actions are required to remove those violations and hence, to postpone these investments.

To date, distributed generators seldom provide voltage support because DSOs normally request them to operate under fixed power factors [1], [2]. According to [3], there are two main reasons why the DGs are not allowed to control voltage: one is that it can destabilize the automatic LTC controller and the other is that, for voltage regulation, it may need high reactive currents and this may overload the equipment.

Methods to better control the existing resources have been proposed in the literature [3]-[5]. The common assumption is that dispersed sources will modulate their production to support the network controllability and flexibility. For example, a centralized controller based on sensitivity analysis is presented in [3]. The optimization problem minimizes the active power curtailment of the DG unit production to regulate the voltages of the network. In [5], the authors propose an intra-day scheduler to adjust the DG power output in order

G. Valverde (e-mail:gvalverde@ieee.org) is with the Dept. of Electrical Engineering and Computer Science of the University of Liège, Sart Tilman B37, B-4000 Liège, Belgium.

T. Van Cutsem (e-mail:t.vancutsem@ulg.ac.be) is with the Fund for Scientific Research (FNRS) at the same department. to minimize the deviation of the control variables and bus voltages with respect to a day-ahead scheduler.

The aforementioned controllers formulate the optimization problem for a single step solution. In addition, they use sensitivity matrices to approximate the change of voltages and power flows with respect to changes of control variables. However, these sensitivity matrices suffer from inaccuracies because they should incorporate the variation of load powers with voltage, which is not well known in practice.

A robust corrective controller must account for these uncertainties. It must also consider the dynamic transition from the measured to the target values, which cannot be achieved from single step optimizations.

A multi-step optimization based on the principles of Model Predictive Control (MPC) is appealing to deal with those issues [6], [7]. MPC has been recently studied as an alternative for controlling power networks with renewable power sources. For example, a control scheme for load management is proposed in [8]. To make the electricity demand more flexible, the aim is to control the demand of residential heaters such that the customers benefit of lower electricity prices. In [9], the author presents an MPC-based control strategy to balance the intermittency of renewable generation by little adjustments of conventional power sources, and few actions in load control by optimally using the storage devices.

The work in [10] takes the principle of transmission Secondary Voltage Control and applies it to distribution networks. The centralized control consists of the correction of the reactive power set-point of each DG unit to correct the pilot bus voltages. Each generator produces a reactive power proportional to its available reserve. This idea was later extended in [11]. An initial Optimal Power Flow (OPF) is used to calculate the voltage references of pilot buses in order to minimize losses, and deals with transformer LTCs.

This paper proposes a corrective control based on MPC to correct voltages out of limits by applying optimal changes of the control variables (mainly active and reactive power of distributed generation and LTC voltage set-point). The key contributions of this paper are listed below:

- The proposed control scheme regulates the network voltages such that they fall within an acceptable range of operation. Hence, the controller does not act unless these limits are violated.

- The controller discriminates between cheap and expensive control actions and selects the appropriate set of control variables depending on the regions of operation also defined in this paper.

- Being based on multiple time step optimization, the 


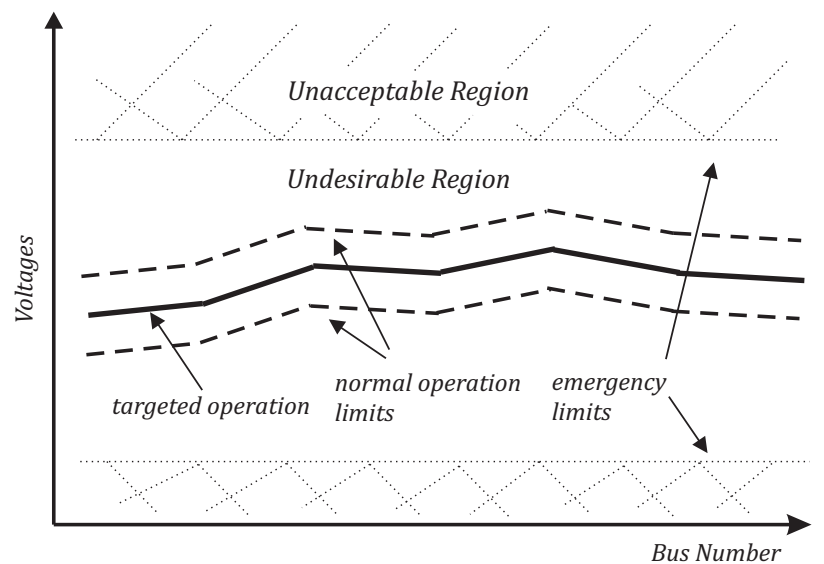

Figure 1. Regions of operation defined by operation and emergency limits

proposed controller is able to smoothly drive the system from its current to the targeted operation region.

- Due to the closed-loop nature of MPC, the proposed control scheme can account for model inaccuracies and failure or delays of the control actions. This is a key feature missing in previous control schemes.

- Lastly, owing to its anticipation capabilities, the proposed controller also takes into consideration the requested actions that will be applied in the future. This is a common situation when the LTC of the transformer is requested to operate at time $k$ but acts later due to standard control delays. Accounting for future control actions avoids the premature and maybe unnecessary dispatch of other control actions.

This paper is organized as follows: Section II introduces the control objective while Section III presents the MPCinspired algorithm to reach that objective. This algorithm is improved in Section IV to predict the effect of future LTC actions on the controlled voltages. Section V describes the 75bus system model used in the tests and outlines the algorithm implementation. Results and conclusions are presented in Sections VI and VII, respectively.

\section{Control Objectives}

Because of higher penetration levels of distributed generation, and due to the massive energy these sources are expected to provide, temporary voltage problems will be encountered in the hosting distribution networks.

The control objective proposed in this paper consists of maintaining the voltages within some predefined limits, as presented in Fig. 1. Initially, the operator will define a target voltage for each bus in the network. This target voltage may follow a security or economical purpose, e.g. network losses minimization. However, trying to reach the actual target values is impractical and likely infeasible. Alternatively, one can try to keep the network voltages within some limits around the target values. In the sequel, these limits are referred to as normal operation limits, and the operation within these limits is the controller's ultimate objective.

If some of the voltages fall in the undesirable region, the controller will use the minimum control actions to bring these

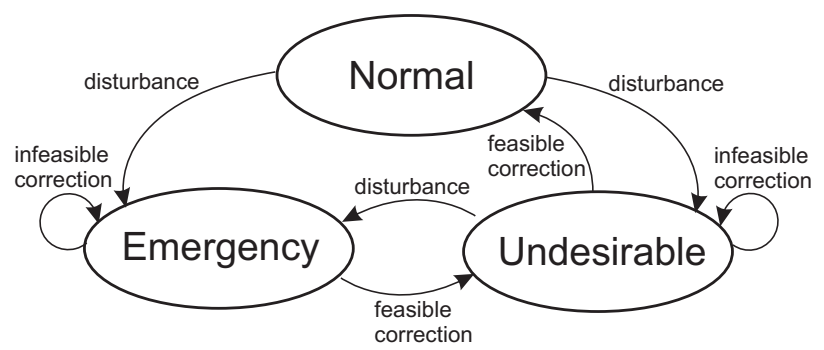

Figure 2. Operation states and corrective actions

voltages within the acceptable limits. As the voltages are in the undesirable region, not in emergency yet, the controller will only use the cheapest control variables, since it is not economically justifiable to use expensive control variables to maintain voltages in a narrow band of operation. To give an example, the requested band of operation for a monitored bus voltage may be set at [1.00 1.02] pu. However, since the targeted operation could be the result of an OPF, the same band does not necessarily apply to all buses, as suggested in Fig. 1. Moreover, the bandwidth for each controlled bus may depend on the importance of the customers connected to this bus or the cost associated with regulating the voltages within a narrow band of operation.

If some bus voltages operate in the unacceptable region, outside of the emergency limits, the controller will use all of the available (cheap and expensive) control variables until the undesirable region is reached, as presented in Fig. 2. The emergency limits can be set the same for all the bus voltages. For instance, the operator can define that the network is under emergency conditions if any of the monitored voltages fall outside the range [0.94 1.06] pu. In practice, these limits will be defined by the corresponding Grid code.

Once the controller succeeds to bring the voltages within the undesirable region, the controller will use again the cheapest control variables to reach the normal operation conditions. The corresponding control variables used in the undesirable and unacceptable regions of operation will be presented in Section III-A.

Fig. 2 summarizes the transition between operation states after disturbances, and the corresponding corrective actions. Note that there are cases where the correction of some bus voltages is infeasible with the available control variables. Under these circumstances, the controller should, at least, apply the control actions that can bring the problematic voltages to a better operation point, even when this point is outside the normal operation limits. The controller must do so until a feasible correction is found.

Finally it is worth mentioning that, as for any other power system security and control scheme, there is a cost for voltage regulation. In the proposed scheme this cost is mainly associated with the difference between the actual power output and the originally scheduled output of DG units. In a way to minimize this cost, the proposed controller minimizes the changes of the control variables when the voltages fall outside the acceptable limits. However, any cost incurred for voltage regulation will be compensated by a more robust distribution 


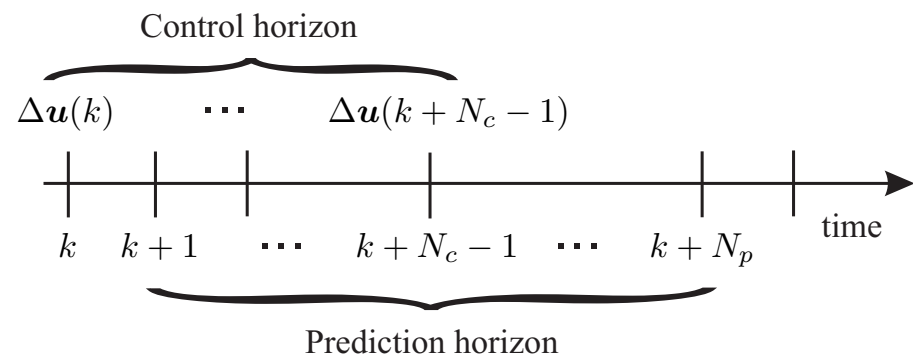

Figure 3. Basic concept of multi-step optimization with horizons

network able to allocate more distributed energy sources.

\section{Multi-Step Corrective Control}

The multi-step optimization problem considered in MPC finds a sequence of control actions in $N_{c}$ steps and predicts the response of these actions in $N_{p}$ steps.

Figure 3 recalls the basic concept of MPC. At instant $k$, using the latest available measurements, the controller determines the optimal change of control variables from $k$ until $k+N_{c}-1$, in order to meet a target at the end of the prediction horizon i.e. at $k+N_{p}$. However, only $\Delta \boldsymbol{u}(k)$ is applied. Then, the controller is updated for $k+1$ with the new set of measurements that reflect the system response to the applied control actions at and before $k$ [12]. Note that the control and prediction horizon are receding.

As presented in Fig. 3, the prediction horizon must be chosen such that it takes into account the expected effect of the computed control actions in the system. Based on this, the length of the prediction horizon should be at least equal to the length of the control horizon, i.e. $N_{p} \geq N_{c}$. From a computational viewpoint, the lengths should be equal unless the controller is requested to consider changes happening beyond the control horizon.

The above principle is applied to voltage control of distribution networks as follows. The control scheme calculates the change of power output of the distributed energy sources $\left(\boldsymbol{P}_{g}, \boldsymbol{Q}_{g}\right)$, and the voltage set-point of the LTC transformer $\left(V_{t a p}\right)$ at the bulk power supply point to maintain the monitored voltages within permissible limits. The change of control variables at time $k$ are:

$$
\Delta \boldsymbol{u}(k)=\left[\Delta \boldsymbol{P}_{g}(k)^{T}, \Delta \boldsymbol{Q}_{g}(k)^{T}, \Delta V_{t a p}(k)\right]^{T}
$$

with $\Delta \boldsymbol{u}(k)=\boldsymbol{u}(k)-\boldsymbol{u}(k-1)$, and where ${ }^{T}$ denotes array transposition.

The overall objective is to minimize the changes of control variables while satisfying voltage limits. This leads to the following standard Quadratic Programming (QP) problem:

$$
\min \sum_{i=0}^{N_{c}-1}\|\Delta \boldsymbol{u}(k+i)\|_{\boldsymbol{R}}^{2}
$$

subject to:

$$
\begin{aligned}
\boldsymbol{u}^{\text {min }} & \leq \boldsymbol{u}(k+i) \leq \boldsymbol{u}^{\text {max }} \\
\Delta \boldsymbol{u}^{\text {min }} & \leq \Delta \boldsymbol{u}(k+i) \leq \Delta \boldsymbol{u}^{\text {max }}
\end{aligned}
$$

$$
\begin{gathered}
\text { for } i=0,1, \ldots, N_{c}-1 . \\
\qquad \mathbf{V}^{\text {min }} \leq \boldsymbol{V}(k+i \mid k) \leq \boldsymbol{V}^{\text {max }} \\
\boldsymbol{V}(k+i \mid k)=\boldsymbol{V}(k+i-1 \mid k)+\frac{\partial \boldsymbol{V}}{\partial \boldsymbol{u}} \Delta \boldsymbol{u}(k+i-1)
\end{gathered}
$$

for $i=1, \ldots, N_{p}$. Here $N_{p} \geq N_{c}$ and $\boldsymbol{R}$ is a weight matrix used to penalize the "expensive" control variables $\boldsymbol{u}$. The changes of the control variables are zero at and beyond the control horizon, i.e. $\Delta \boldsymbol{u}(k+i)=\mathbf{0}$ for $i \geq N_{c}$. Finally, $\boldsymbol{V}(k+i \mid k)$ is the set of predicted bus voltage magnitudes given the measurements at time instant $k$ and $\frac{\partial V}{\partial \boldsymbol{u}}$ is the sensitivity matrix of bus voltages with respect to the control variables.

The controller is updated by the real-time measurements of bus voltage magnitude $\boldsymbol{V}(k \mid k)$, and the previous control signals $\boldsymbol{u}(k-1)$.

\section{A. Weighting and selection of control variables}

The weight assigned to each control variable is directly related to the cost of the device to provide ancillary services. For example, the reactive power output of the DG units and the LTC voltage set-point are considered cheap controls.

The active power output of a DG unit is considered an expensive control and should not be requested unless emergency conditions are encountered. As presented in Fig. 1, if some of the voltages are outside the emergency constraints, the controller will use all controls to correct them. Thus, active powers of the DG units should be heavily weighted to minimize their use. Once the voltages reach the undesirable region, only the reactive powers and the voltage set-point of the LTC will be used as control variables.

\section{B. Limits on control variables}

The active power outputs of DG units are constrained by their capacity. For example, the active power production of conventional synchronous machines is constrained by the turbine capacity. In renewable energy sources, where the production is driven by weather conditions, the corresponding variables of active power are upper bounded by the actual power extracted from the wind or the sun. This is, at any instant $k$, the controller cannot request more than the power that is being produced, but it can request active power reductions by partial curtailment. On the other hand, the reactive power output of renewable energy sources is considered fully controllable but subject to capacity limits.

The reactive power injection of synchronous machines is constrained by the maximum allowed armature and field currents while power absorption is constrained by stability, as well as by armature core end heating [13].

Although the maximum reactive power production can be fixed for each DG unit, it is desirable to update it with the actual terminal voltage and active power production [14], so that full advantage is taken from its capability. This information will be used then to update the limits on the control variables, presented in (2a).

The constraints (2b) can be used to protect the machines and inverters against fast ramping (up or down) and make 
the controller decide more cautiously of how to distribute the available control actions. For example, in order to avoid overusing the transformer tap changer, the LTC set-point changes could be limited (up or down), for instance to no more than half the LTC voltage dead band, or even less.

\section{Variation of voltage constraints in the prediction horizon}

The controller must eventually bring the controlled voltages inside the limits defined by the normal operation constraints (see Fig. 1). One option is to have those constraints enforced at each time step, as in (2c), but this may lead to large and unnecessary changes of the control variables to meet $(2 \mathrm{c})$. Another option is to have them enforced at the end of the prediction horizon only, but this may lead to slow voltage correction. The compromise considered in this paper consists of making a progressive tightening of the voltage limits $\boldsymbol{V}^{\text {min }}$ and $\boldsymbol{V}^{\max }$ in the horizon. Therefore, a linear variation has been considered in this paper:

$$
\mathbf{V}^{\min }(k+i) \leq \boldsymbol{V}(k+i \mid k) \leq \boldsymbol{V}^{\max }(k+i)
$$

where,

$$
\begin{aligned}
& \mathbf{V}^{\min }(k+i)=\left(1-\frac{N_{p}-i}{\rho N_{p}}\right) \boldsymbol{V}^{\text {min }} \\
& \mathbf{V}^{\max }(k+i)=\left(1+\frac{N_{p}-i}{\rho N_{p}}\right) \boldsymbol{V}^{\max }
\end{aligned}
$$

for $i=1, \ldots, N_{p}$ and $\rho$ is a tuning parameter used to modulate the rate of change of the voltage limits in the horizon.

\section{Relaxation of voltage constraints}

Under some circumstances, the available controls may not be enough to bring voltages within the normal operation constraints, i.e. the optimization in (2) is infeasible. This may happen under the following conditions:

1) The voltage constraints are too strict so that it is impossible to operate with all voltages within a narrow band;

2) the DG units reach their maximum or minimum power outputs, or

3) after a disturbance, the new operation point is very far from the desired voltage values.

Clearly, it is unacceptable for the controller to output an infeasible solution, particularly during emergency conditions when it is mostly needed. Instead, the multi-step optimization should determine the sequence of actions that will bring the problematic voltages as closely as possible to the desired values. To do so, the optimization problem defined in (2) is extended to include some slack values that can relax the constraints. Note that relaxation is applied to the voltage constraints (2c) only. It cannot be applied to (2a)-(2b), since the controller must not violate the capacity limits of the generators, inverters and the LTC. Furthermore, constraint relaxation should not be used unless strictly necessary. Thus, voltage constraints are relaxed if the available control variables are not enough to reach the desired voltages.

The objective in (2) is modified as follows:

$$
\min \sum_{i=0}^{N_{c}-1}\|\Delta \boldsymbol{u}(k+i)\|_{\boldsymbol{R}}^{2}+\|\varepsilon\|_{\boldsymbol{S}}^{2}
$$

while constraint $(2 \mathrm{c})$ is replaced by:

$$
-\varepsilon_{1} \mathbf{1}+\mathbf{V}^{\min }(k+i) \leq \boldsymbol{V}(k+i \mid k) \leq \boldsymbol{V}^{\max }(k+i)+\varepsilon_{2} \mathbf{1}
$$

where $i=1,2, \ldots, N_{p}, \mathbf{1}$ denotes a unitary vector, and $\varepsilon=$ $\left[\varepsilon_{1}, \varepsilon_{2}\right]^{T}$ is the vector of slack variables. The latter are heavily penalized in the cost function using the weight matrix $S$, so that there is a strong incentive to keep them at zero whenever possible [6].

An alternative formulation would consist of using a slack variable for each voltage constraint, and each prediction step. This would automatically point out the problematic voltage constraints as those with a nonzero $\varepsilon_{j}$ value. However, this is achieved at the expense of larger computational effort and time since many slack variables would have to be processed.

\section{E. Sensitivity matrix calculation}

An accurate sensitivity matrix should incorporate the variation of load powers with voltage, the actual network impedances and the actual system operation point. Unfortunately, this information is not well known in practice and some approximations are required.

The sensitivity of bus voltages with respect to power injections can be obtained from the inverse of the Jacobian matrix extracted from an offline power flow calculation [3], [5].

The sensitivities of bus voltages with respect to the voltage set-point can be approximated by computing the ratio of variations of the monitored bus voltages to the LTC's controlled bus voltage due to a tap change. This information can be easily extracted from the solution of two power flow runs with a single tap position difference. These calculations are also made offline and the sensitivity matrix in (2d) is seldom updated depending on the network operation, e.g. heavy or light loading conditions. It is expected that the MPC will compensate for infrequently updated sensitivities.

\section{INCLUSION OF FUTURE LTC ACTIONS IN CONTROLLER}

An LTC is a slowly acting device that controls the distribution side voltage of the transformer. The LTC performs a tap change if the controlled voltage remains outside of a deadband for longer than a predefined delay [14]. This delay is specified to avoid frequent and unnecessary tap changes that may reduce the LTC lifetime. If more than one step is required, the LTC will move by one step at a time with delay between successive moves.

The proposed controller leaves this local control unchanged but acts on the LTC set-point $V_{t a p}$ if appropriate. Therefore, tap changes will be triggered when changes in operation conditions push the controlled voltage $V_{2}$ out of the deadband, or when the controller requests a change of $V_{t a p}$ (and, hence, a shift of the dead-band) such that the distribution side voltage of the transformer falls outside the dead-band. 
Control horizon

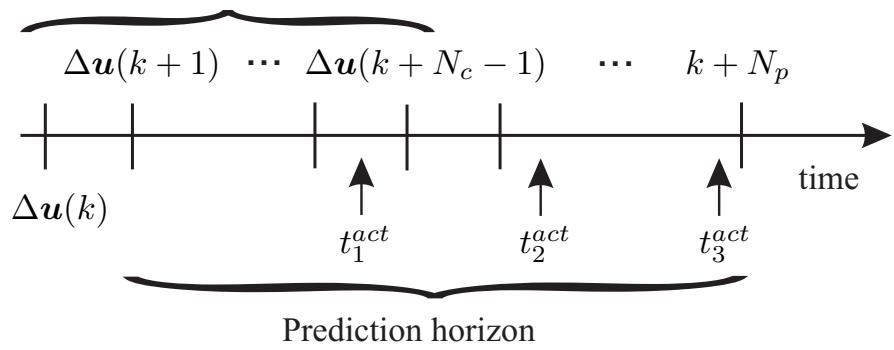

Figure 4. Extension of prediction horizon to include predicted LTC actions

By assuming that a tap change produces $\Delta V_{d}$ variation in $V_{2}$, the number of tap changes can be roughly estimated by:

$$
N_{o p}= \begin{cases}\operatorname{roundup}\left[\frac{V_{2}-V_{t a p}-d}{\Delta V_{d}}\right], & \text { if } V_{2}>V_{t a p}+d \\ \operatorname{roundup}\left[\frac{V_{t a p}-d-V_{2}}{\Delta V_{d}}\right], & \text { if } V_{2}<V_{t a p}-d\end{cases}
$$

where $d$ is half the LTC dead-band and the function roundup provides the nearest upper integer. Note that $\Delta V_{d}$ is measurable after an LTC action occurs. This value is assumed constant and it is considered a known value for the controller.

From (7), the times of LTC actions can be estimated as:

$$
t_{j}^{a c t}=t_{k}+T_{f 0}+T_{f}(j-1)
$$

for $j=1, \ldots, N_{o p}$. Here, $t_{k}$ is the present time instant, $T_{f 0}$ is the time delay for the first step and $T_{f}$ is the time delay for subsequent tap steps. The controller can use this information to anticipate the future voltage changes due to the operation of the LTC. In order to do so, the controller must extend the prediction horizon $N_{p}$ until the last predicted LTC control action is included. A general example of this is provided in Fig. 4. At instant $k$, it is predicted from (7) and (8) that three tap changes will take place at $t_{1}^{a c t}, t_{2}^{a c t}$ and $t_{3}^{a c t}$, respectively, with $t_{2}^{a c t}$ and $t_{3}^{a c t}$ beyond the control horizon. In order to account for all these LTC actions, the controller extends the prediction horizon up to the smallest discrete time larger than $t_{3}^{\text {act }}$.

The effect of the voltage changes is estimated given the sensitivities of bus voltages to the secondary voltage of the main transformer, as explained in Section III-E. Furthermore, the tap position changes are handled as known disturbances, accounted for by replacing (2d) by:

$$
\begin{aligned}
\boldsymbol{V}(k+i \mid k)= & \boldsymbol{V}(k+i-1 \mid k)+\frac{\partial \boldsymbol{V}}{\partial \boldsymbol{u}} \Delta \boldsymbol{u}(k+i-1) \\
& +\frac{\partial \boldsymbol{V}}{\partial V_{\text {tap }}} \Delta V_{d} \gamma(k+i)
\end{aligned}
$$

with $i=1, \ldots, N_{p}$ and $\gamma$ is a binary variable equal to one for the instants when the tap changes have been predicted, or zero otherwise. Additionally, $\frac{\partial \boldsymbol{V}}{\partial V_{t a p}}$ is the last column of matrix $\frac{\partial \boldsymbol{V}}{\partial \boldsymbol{u}}$.

With the controller being able to predict the effect of future LTC actions, it is possible to avoid premature and unnecessary output changes of DG units since it can better decide whether or not the cheap LTC actions are enough to correct the controlled voltages.

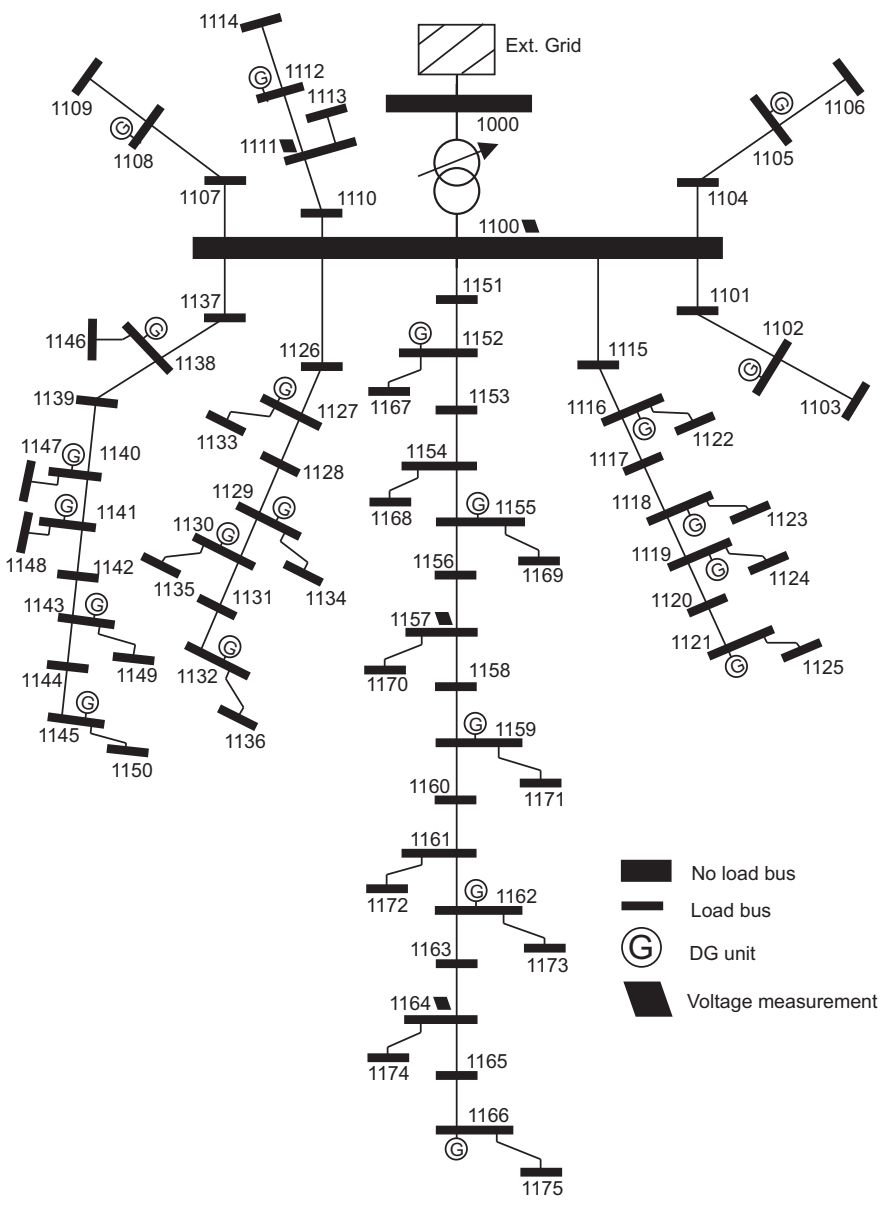

Figure 5. Network topology and measurement allocation

To summarize, the final control scheme proposed in this paper consists of minimizing (6) subject to (2a), (2b), (6a) and (9).

\section{Test System And Algorithm IMPlementation}

The multi-step receding-horizon controller presented in Sections III and IV has been tested through simulations of a 75bus, $11-\mathrm{kV}$ radial distribution network hosting 22 DG units; the line parameters are available in [15] and the network topology is shown in Fig. 5.

The distribution system is connected to the external grid through a $33 / 11 \mathrm{kV}$ transformer equipped with LTC. The network topology consists of eight feeders all directly connected to the main transformer and they serve 38 loads modeled as constant current for active power and constant impedance for reactive power, and 15 more loads represented by equivalent induction motors.

This system and the proposed controller have been simulated in Matlab/SIMULINK with detailed models of static and dynamic loads, and the distributed generators with their respective local controllers. These models are part of the library developed in [16]. The controller was implemented in SIMULINK through an S-function block resorting to the QP solver SQOPT developed by TOMLAB [17]. 


\section{A. Operation of $D G$ units}

In this test system, 13 out of the 22 DG units are 3MVA synchronous generators driven by hydro turbines with $2.55 \mathrm{MW}$ of maximum capacity. The active power output of each machine is regulated by a Proportional and Integral (PI) controller in order to meet the demands of the MPCbased controller. Each generator is also controlled by an Automatic Voltage Regulator (AVR) with an inner control loop to regulate the terminal voltage in response to fast changes, and an outer PI control loop to adjust the reactive power to the set-point demanded by the centralized controller. Reference [18] provides a schematic diagram of these control loops. The reactive power loop has a much slower time response compared to the voltage loop. This time-scale separation is needed for stability.

In addition, each synchronous generator is equipped with an Over-Excitation Limiter (OEL) that reduces the field current after some overload duration. The centralized controller limits the reactive power requests to avoid the triggering of the OELs.

The remaining nine DG units are Doubly-Fed Induction Generators (DFIG) driven by wind turbines. Each DFIG is a one-machine equivalent of two $1.5 \mathrm{MW}$ wind turbines operating in parallel. The nominal capacity of each DFIG is 3.33 MVA. The model of the wind turbine and its parameters were taken from [19].

The controller can request reduction of the active power output through pitch control of the turbine blades.

The DFIGs operate in reactive power control mode. This is achieved by a PI controller that regulates the reactive power output according to the set-point value requested by the centralized controller. Compared to the synchronous machines, this reactive power control loop has a faster response.

The reactive power limits of DG units are calculated, at any time $k$, given their actual operation conditions $\left(\boldsymbol{P}_{g}\right.$ and $\boldsymbol{V}_{g}$ ) and their nominal capacities. Hence, any reactive power increase requested by the controller will not compromise the DGs active power output or violate the machine capacities.

\section{B. Measurement configuration and data collection}

It is assumed that the 22 DG units are allocated with remote units that measure the active power, reactive power and voltage magnitude at their terminals. In addition, the distribution side voltage of the main transformer is monitored and this information is sent to the centralized controller along with the actual voltage set-point of the LTC.

As regards load buses, the measurement configuration is such that no load is at a distance larger than two buses from a voltage monitored bus, see Fig. 5. By following this rule, there are three load buses with monitored voltages. These voltage measurements along with the power output measurement of the DG units make up a set of 71 measurements received by the controller.

It is not possible to ensure that non-monitored voltages will be within the desirable limits. However, by distributing the measurements all over the network, it is reasonable to expect that the voltages of non-monitored buses will be close to the voltages of the neighboring controlled buses.

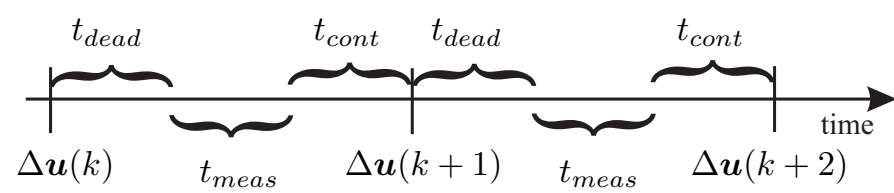

Figure 6. Definition of controller's times between control actions

The measurements are collected some time after the control actions are applied. This is to wait for the system response and to avoid making decisions based on measurements taken during transients.

Because of the fast sampling rate of modern monitoring units and their efficient communication links, it is assumed that the measurements are collected every $0.2 \mathrm{~s}$.

The measurements were simulated by adding white Gaussian noise restricted to $\pm 1 \%$ for $\boldsymbol{V}$ measurements and $\pm 1 \%$ of the respective DG maximum power output for $\boldsymbol{P}_{g}$ and $\boldsymbol{Q}_{g}$ measurements. This noise level is larger than the accuracy specifications of currently available metering devices, reported to be in the order of $\pm 0.1 \%$ of voltage and current readings, see for example [20]. In order to filter out some of this noise, the controller uses the average of the 11 snapshots received over a time window of $2 \mathrm{~s}$.

Figure 6 presents the time sequence of events. At instant $k$, when a control action is applied, the controller waits for a dead time $t_{\text {dead }}$. After this time has passed, the measurement collection and averaging is performed over a time window $t_{\text {meas }}$. Finally, with the averaged measurements available, the controller takes some time $t_{\text {cont }}$ to compute and transmit the new control set-points to the DG units and the LTC. The results reported in this paper were obtained with $t_{\text {dead }}=4 \mathrm{~s}$, $t_{\text {meas }}=2 \mathrm{~s}$, and $t_{\text {cont }}=4 \mathrm{~s}$.

\section{Simulation Results}

For all test cases, it is required that the monitored voltages remain within the $\left[\begin{array}{ll}1.000 & 1.025\end{array}\right] \mathrm{pu}$ range. In addition, the system is considered under emergency conditions when any bus reaches voltages outside the [0.940 1.060] pu interval.

The active and reactive power of DG units are not allowed to change more than $0.5 \mathrm{MW}$ and 0.5 MVAr respectively, while the LTC set-point is not allowed to change more than $0.01 \mathrm{pu}$ for each time of control actions. The system base is 100 MVA.

In the objective function (6), identical costs have been assumed for all DGs. Changes of the reactive power outputs cost the same as changes in LTC voltage set-point while the cost for active power changes is set 10 times higher. Moreover, the cost of using the slack values is 1000 times higher than that of reactive power. Finally, the progressive tightening of the voltage limits in the prediction horizon was tuned with $\rho=5$ and $N_{p}=N_{c}=3$ unless specified.

Since the variation of load powers with voltage are not well known in practice, the sensitivity matrix has been calculated by considering constant power models for all loads. In addition, the line parameters used to calculate the sensitivity matrix were corrupted by a random error whose mean value is zero and standard deviation is $10 \%$ of the actual line parameter. 


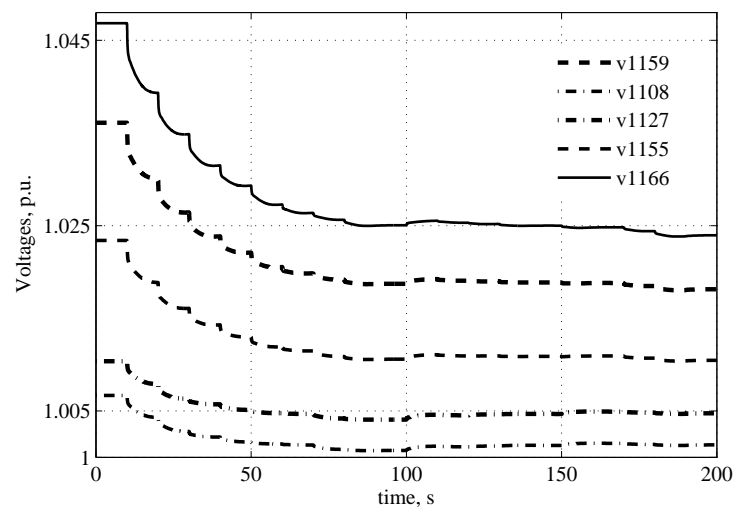

Figure 7. Voltage correction for Scenario A

The objective is to demonstrate that the controller is robust and can compensate for these model inaccuracies.

\section{A. Correction of local voltage problems with DGs}

The first scenario reported consists of the presence of over voltages in certain areas of the network caused by low power demands and high power production from DG units.

With the available set of measurements, it is found that the voltages in some areas are outside the normal operation constraints, but not enough to reach the emergency state.

Here it is pointed out that, for legibility purposes, the following plots show the exact (noiseless) voltages and DG power outputs, as opposed to the noisy and discrete measurements received and processed by the controller.

Figure 7 presents the obtained correction of the voltages starting at $t=10 \mathrm{~s}$ and changing every $10 \mathrm{~s}$ thereafter, under the effect of the controller adjusting the DG reactive powers. Note that the controller yields an exponentially decreasing correction of the bus voltages until the latter reach the desired interval.

The selected curves in Fig. 7, correspond to the most representative voltages in the network. The only load bus voltage reported in this paper is v1159 whereas the remaining voltages correspond to DG buses.

Although most of the DG units reduced their reactive power production, the ones connected to buses 1102 and 1108 were requested to increase, see Fig. 8. This is because the controller anticipated that the voltages near bus 1108 would violate the lower limit of $1 \mathrm{pu}$. Hence, the reactive powers of these machines increase to maintain those voltages within limits.

From Fig. 8 it is seen that the maximum change of reactive power output occurs in the DG unit at bus 1166. This machine is requested to absorb reactive power until the most problematic voltage v1166 is corrected.

As soon as all the monitored voltages are brought back inside the limits, the controller does not request further changes in $\Delta \boldsymbol{u}$. This condition is met at $t=190 \mathrm{~s}$. It is interesting to note that even in the presence of inaccurate sensitivity values, the controller is able to correct the voltages.

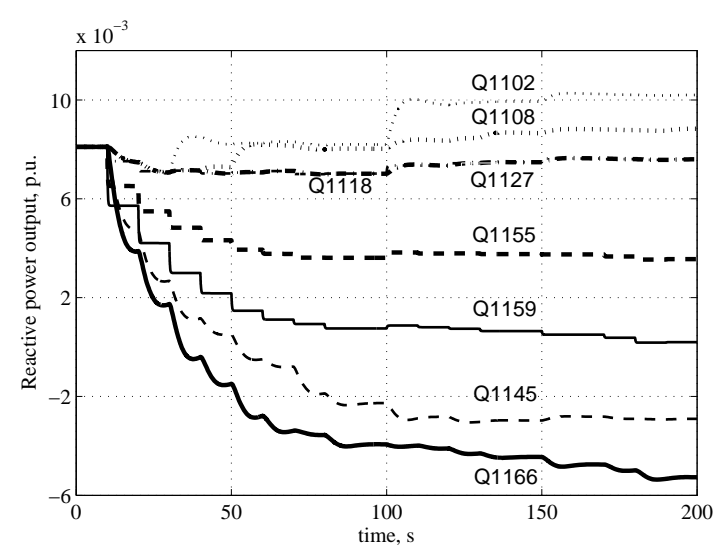

Figure 8. Reactive power output of the DGs for Scenario A

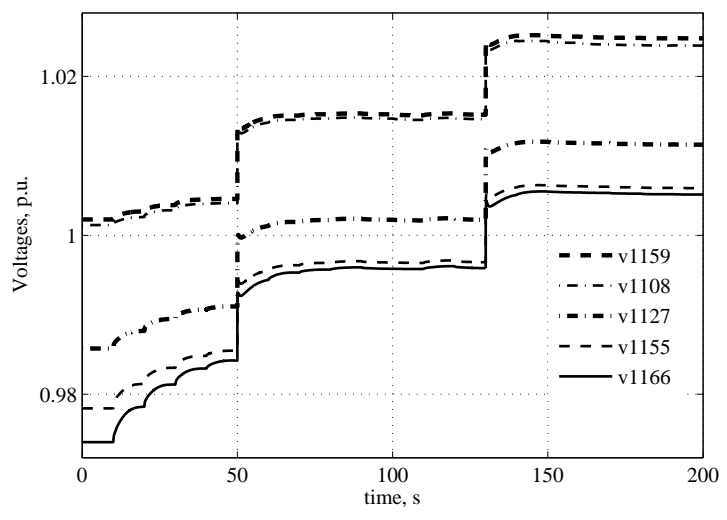

Figure 9. Voltage correction for Scenario B

\section{B. Correction of local voltage problems with DGs and LTC}

In the second scenario, the system operates at peak demand so that some buses are facing low voltage conditions. In this case, the LTC voltage set-point along with the reactive power of DG units is used to correct the low voltage problems. The active power of DG units is not used because the system has not reached the emergency conditions.

Figure 9 presents the performed voltage corrections by optimum changes of the control variables. At $t=130 \mathrm{~s}$, the controller is able to correct the low voltages while making sure that no other voltage reaches the upper limit of $1.025 \mathrm{pu}$. The large discrete events at $t=50 \mathrm{~s}$ and $t=130 \mathrm{~s}$ were produced by the changes in the tap position, triggered by changes of the voltage set-point of the LTC.

Figure 10 shows the voltage set-point ordered by the centralized controller. Although it changes the LTC voltage set-point since $t=10 \mathrm{~s}$, it is at $t=30 \mathrm{~s}$ when the LTC controlled voltage falls outside the allowable dead-band. The LTC has a time delay $T_{f 0}=20 \mathrm{~s}$ for the first tap change and $T_{f}=10 \mathrm{~s}$ for subsequent tap changes.

In the time interval [50 130] s, the controller predicts that by requesting minimum changes of the LTC voltage set-point, it will reach the desired voltages. However, the LTC does not move a new tap change unless the voltage falls outside the voltage dead-band defined in (7). Hence, for this time interval, 


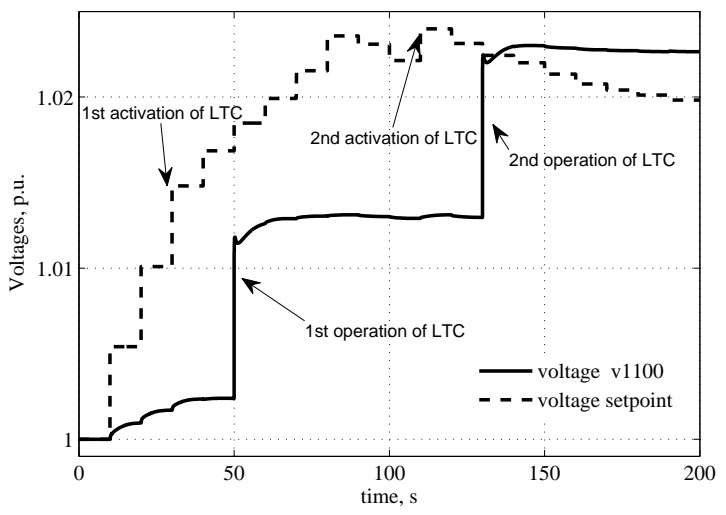

Figure 10. LTC actions for Scenario B

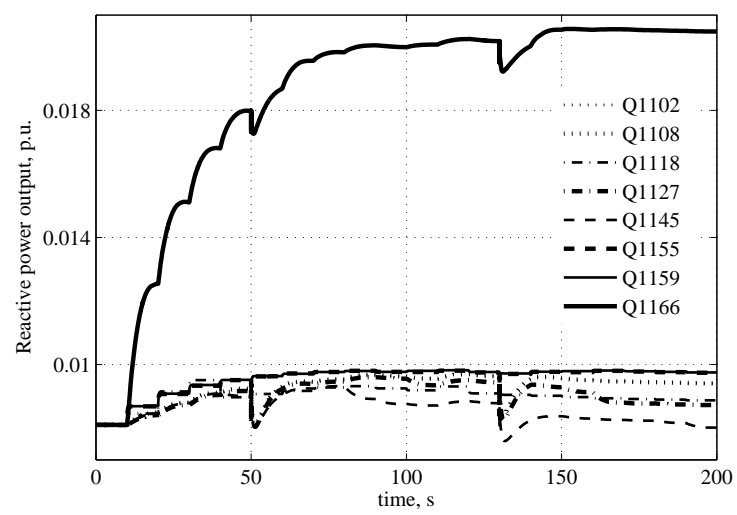

Figure 11. Reactive power output of some DGs for Scenario B

the controller faces a situation where it requests a control action that is not reflected in the network. At $t=110 \mathrm{~s}$, the LTC eventually triggers a new tap change that is performed $20 \mathrm{~s}$ later.

As explained in Section IV, during the computation of the optimal control actions for $t=40,50,120$ and $130 \mathrm{~s}$, the controller knows that a tap change is about to occur, and takes this into account to predict its effect on the voltages. Since the predicted LTC actions occurred within the $N_{p}=3$ steps, there was no need to extend the prediction horizon.

Figure 11 presents the corresponding evolution of some DG reactive power outputs. During the first $50 \mathrm{~s}$, the increase of the reactive powers helps recovering the voltages. For the remaining of the simulated scenario, the LTC was mainly in charge of the voltage correction.

The lowest voltage v1166 is partly corrected by large reactive power production of the DG at bus 1166. This large and isolated effort is explained because no other DG can help recovering this voltage. For example, the DFIG at bus 1159 cannot provide more reactive power because it would bring v1159 above the upper voltage limit.

From Figs. $9-11$, it is interesting to note that for $t>130 \mathrm{~s}$, the controller requests small reductions of the LTC voltage set-point and reactive power outputs in order to maintain the voltages v1159 and v1108 below the upper limit.

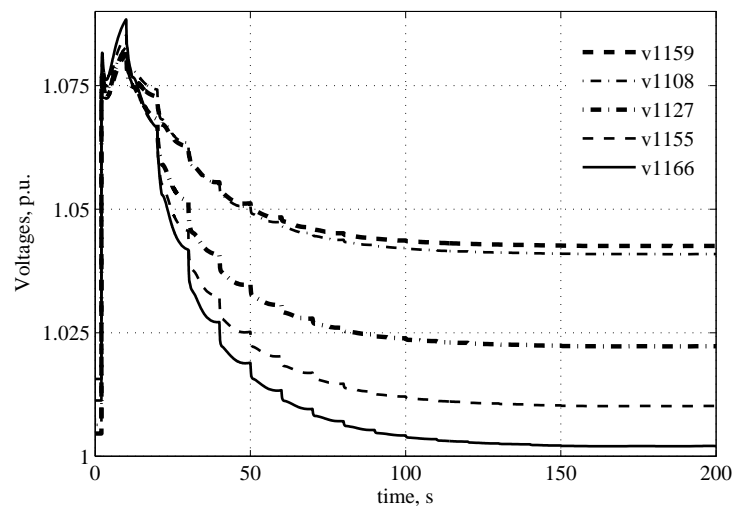

Figure 12. Partial voltage correction for Scenario C

\section{Partial correction of voltages after disturbance - No LTC}

In order to demonstrate the performance of the proposed controller during emergency conditions, the distribution network is affected by a large disturbance in the external grid, namely a step increase of 0.08 pu of the Thévenin equivalent voltage (at the primary side of the main transformer). This causes an increase of the voltage at the connection point which affects all distribution voltages as well. Furthermore, for this test, it is assumed that the LTC of the main transformer cannot operate. The purpose is to check the performance of the controller during emergency conditions and with limited control capability.

Figure 12 shows the partial correction of voltages after the disturbance that takes place at $t=3 \mathrm{~s}$. Due to the severity of the disturbance, some voltages in the network reach the emergency $1.06 \mathrm{pu}$ upper limit. In order to solve this problem, the controller makes use of the active and reactive power outputs of all the DG units. Once the voltages are brought back to non-emergency conditions, the controller only uses the reactive power outputs.

As can be seen from Fig. 13, the DG units reach their minimum reactive power. Therefore, the controller does not succeed to fully correct the voltages. In fact, by lack of control means, the voltage constraints have been relaxed to get a feasible solution. In average, the controller had to soften the upper voltage limits by $0.019 \mathrm{pu}$. This is approximately the difference between the final voltage at bus 1159 and the voltage limit of $1.025 \mathrm{pu}$.

Figure 14 presents the active power outputs of some DG units. It can be seen that the controller makes use of these control variables while some voltages are under emergency conditions, namely for $t<30 \mathrm{~s}$. When it detects that all monitored voltages are outside the emergency region, at $t=35 \mathrm{~s}$ approximately, the controller stops using the active powers as control variables.

As demonstrated in this test case, the use of slack values can provide partial correction of the voltages in case the optimization problem is infeasible. However, if the emergency conditions were such that the controller decisions aggravate the state of emergency, the controller would be taken out of operation. 


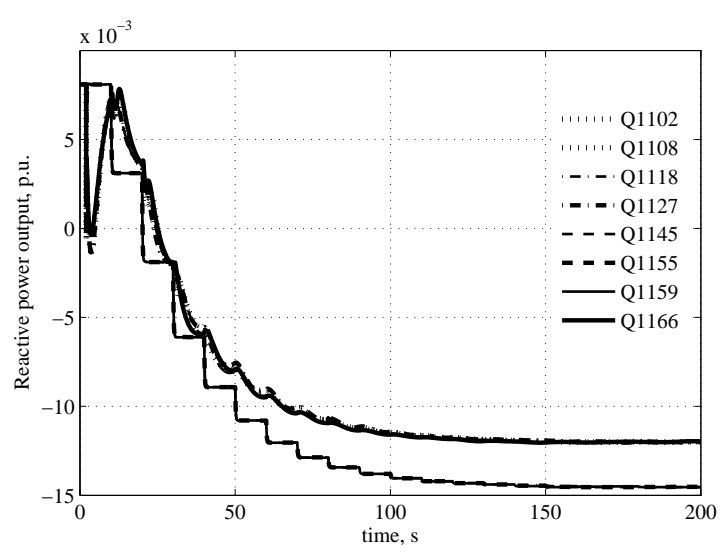

Figure 13. Reactive power output of the DGs for Scenario C

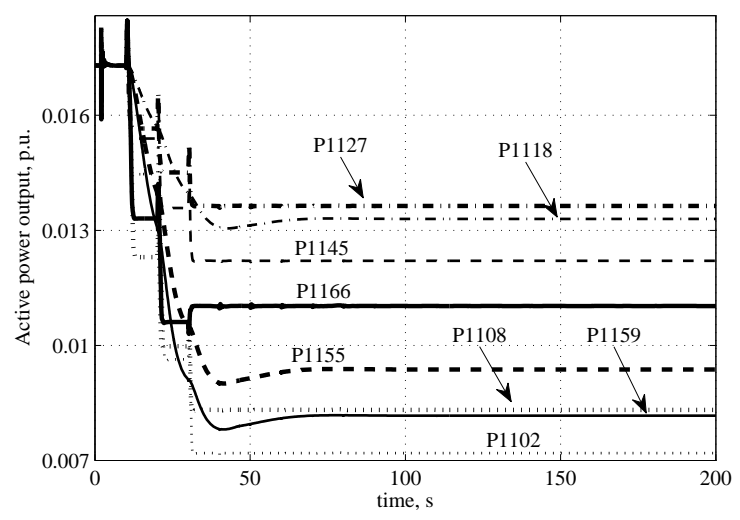

Figure 14. Active power output of the DGs for Scenario C

\section{Correction of voltages with LTC as control variable during emergency conditions}

The last scenario reported consists of an external voltage drop that affects the voltages at all buses. This disturbance was simulated by a negative step change of 0.08 pu of the Thévenin equivalent voltage, at the primary side of the main transformer.

For this case, the LTC is coordinated along with the DG units to correct the voltage drop in the distribution network. The purpose of this test is to demonstrate the prediction capability of the proposed controller and how this avoids unnecessary changes of alternative controls.

Figure 15 presents the voltage correction at a sample of monitored buses. The disturbance was corrected in about $90 \mathrm{~s}$ after the operation of the LTC and few changes of the DG power outputs.

Just after the disturbance at $t=1 \mathrm{~s}$, the difference between the monitored voltage and its set-point is enough to trigger eight tap movements. The first tap operation occurs at $t=21 \mathrm{~s}$ and the subsequent tap movements occur in steps of $10 \mathrm{~s}$ until $t=91 \mathrm{~s}$. After this time, no more LTC actions are required.

The tiny voltage corrections seen mainly at bus 1166 at $t=130 \mathrm{~s}$ and $t=160 \mathrm{~s}$ were triggered by the noise affecting voltage measurements that indicated small violations of the monitored voltages.

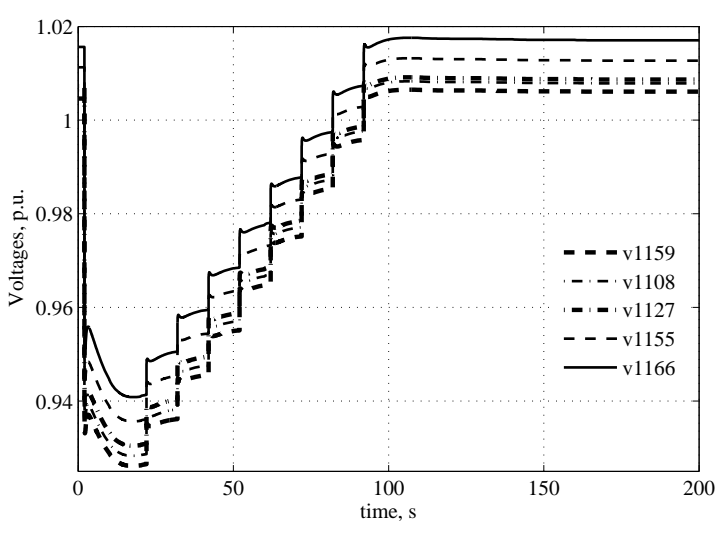

Figure 15. Voltage correction for Scenario D

Table I

Controller Anticipation of The LTC ACtions

\begin{tabular}{cccccccccc}
\hline \hline$t[s]$ & $N_{o p}$ & $N_{p}$ & $t_{1}$ & $t_{2}$ & $t_{3}$ & $t_{4}$ & $t_{5}$ & $t_{6}$ & $t_{7}$ \\
\hline 10 & 7 & 9 & 30 & 40 & 50 & 60 & 70 & 80 & 90 \\
20 & 7 & 8 & 30 & 40 & 50 & 60 & 70 & 80 & 90 \\
30 & 7 & 7 & 30 & 40 & 50 & 60 & 70 & 80 & 90 \\
40 & 6 & 6 & 40 & 50 & 60 & 70 & 80 & 90 & - \\
50 & 5 & 5 & 50 & 60 & 70 & 80 & 90 & - & - \\
60 & 3 & 3 & 60 & 70 & 80 & - & - & - & - \\
70 & 3 & 3 & 70 & 80 & 90 & - & - & - & - \\
80 & 2 & 3 & 80 & 90 & - & - & - & - & - \\
90 & 1 & 3 & 90 & - & - & - & - & - & - \\
\hline \hline
\end{tabular}

Table I details the future tap movements as anticipated by the controller. Equations (7) and (8) are used for this purpose. It is found that the LTC will operate seven times. The controller makes a rough estimate of the LTC time actions. For example, the controller wrongly anticipated that the first LTC action would occur at $t=30 \mathrm{~s}$, while it actually occurred at $t=21 \mathrm{~s}$. This example confirms that there is no need to know the exact moment at which the LTC will act. In fact, any mistake of prediction will be corrected and updated when new measurements are available. The important aspect is that the controller can anticipate future actions and use this information to avoid more expensive control actions.

In order to capture all the anticipated future events due to LTC actions, the controller extended its prediction horizon length as required. For example, at $t=10 \mathrm{~s}$, the controller anticipated that the last control action would occur at $t=90 \mathrm{~s}$. Hence, the controller automatically increases $N_{p}$ from three to nine steps. For $t>50 \mathrm{~s}, N_{p}$ is reset to three.

Figure 16 presents the coordinated reactive power output of the DG units. Since the controller is able to predict the effect of the LTC's future actions in the controlled voltages, the controller did not require significant changes of $\boldsymbol{Q}_{g}$. A similar behavior occurs for changes in active power outputs during the emergency conditions.

For the controller, the cost of staying idle is zero. One could argue that there is a cost associated with many tap movements due to wear of equipment. This could be included in the cost function to reduce the number of tap changes by making use of the other available control variables.

In emergency conditions, the transmission system operator 


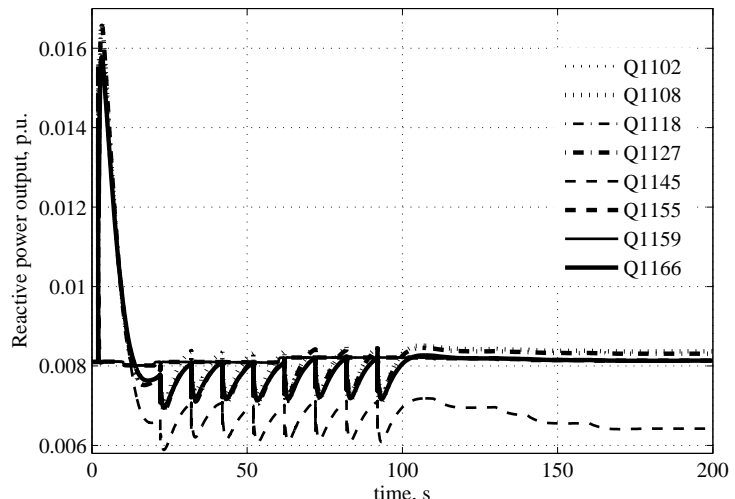

Figure 16. Reactive power output of the DGs for Scenario D

may request to either block the LTC or reduce its set-point (e.g. by $5 \%$ ) in order to take advantage of load dependency on voltage. In such cases, the LTC voltage set-point is no longer a control variable for the MPC controller. However, the tap changes that will take place as a result of that emergency action are taken into account in the proposed scheme as external disturbances, as explained in Section IV.

In terms of execution times, the average CPU time required by the controller was $0.1 \mathrm{~s}$ where half of it was used by the QP solver $S Q O P T$, for $N_{c}=N_{p}=3$. In the worst case, when the prediction horizon was extended to $N_{p}=8$, the CPU time was $0.16 \mathrm{~s}$ where $0.09 \mathrm{~s}$ were used by the QP solver. These times were obtained with SIMULINK using an i7-2620M CPU @ 2.70 GHz laptop.

\section{CONCLUSIONS}

A centralized controller based on MPC has been proposed in this paper for the purpose of voltage control in distribution networks. The controller coordinates the power output of DG units and the voltage set-point of the LTC to smoothly bring the unacceptable voltages back within their limits.

It was found that the controller is able to regulate the monitored voltages during extreme operation conditions and after external disturbances. This was achieved while using inaccurate sensitivity matrices and noisy measurements.

The robustness of the controller was enhanced by including slack variables to relax the voltage constraints in case of optimization infeasibility. This helped to identify the sequence of control actions needed to improve the most problematic voltages, even when the problem is infeasible with the initial constraints. Finally, the controller's prediction skills were demonstrated. The controller anticipates the effect of future actions of the LTC. By doing so, it avoids temporary and unnecessary changes of the more expensive DG power outputs.

\section{REFERENCES}

[1] M.J. Dolan, E.M. Davidson, I. Kockar, G.W. Ault and S.D.J. McArthur, "Distribution power flow management utilizing an online optimal power flow technique," IEEE Transactions on Power Systems, vol. 27, no. 2. pp. 790-799, May 2012.

[2] A.G. Madureira and J.A. Pecas Lopes, "Coordinated voltage support in distribution networks with distributed generation and microgrids," IET Renewable Power Generation, vol. 3, no.4, pp. 439-454, Dec. 2009.
[3] Q. Zhou and J.W. Bialek, "Generation curtailment to manage voltage constraints in distribution networks," IET Generation Transmission and Distribution, vol. 1, no. 3, pp. 492-498. May 2007.

[4] M. E. Baran and I. M. El-Markabi, "A multiagent-based dispatching scheme for distributed generators for voltage support on distribution feeders," IEEE Transactions on Power Systems, vol. 22, no. 1, pp. 52-59, Feb. 2007.

[5] A. Borghetti, M. Bosetti, S. Grillo, S. Massucco, C.A. Nucci, M. Paolone, and F. Silvestro, "Short-term scheduling and control of active distribution systems with high penetration of renewable resources," IEEE Systems Journal, vol. 4, no. 3, pp. 313-322, Sept. 2010.

[6] J. M. Maciejowski, Predictive Control With Constraints. Englewood Cliffs, NJ: Prentice-Hall, 2002.

[7] M. Glavic, M. Hajian, W. Rosehart and T. Van Cutsem, "Recedinghorizon multi-step optimization to correct nonviable or unstable transmission voltages," IEEE Transactions on Power Systems, vol. 26, no. 3, pp. 1641 - 1650, Aug. 2011.

[8] Z. Yi, D. Kullmann, A. Thavlov, O. Gehrke, and H.W. Bindner, "Application of model predictive control for active load management in a distributed power system with high wind penetration," IEEE Transactions on Smart Grids, vol.3, no.2, pp.1055-1062, Jun. 2012

[9] G. Hug-Glanzmann, "Coordination of intermittent generation with storage, demand control and conventional energy sources," IREP Symposium: Bulk Power System Dynamics and Control, Buzios, RJ, Brazil, 2010.

[10] O. Richardot, A. Viciu, Y. Bésanger, N. Hadjsaïd, and C. Kieny, "Coordinated voltage control in distribution networks using distributed generation," IEEE PES Transmission and Distribution Conference and Exhibition, pp.1196-1201, May 2006.

[11] M. Biserica, B. Berseneff, Y. Besanger, and C. Kieny, "Upgraded coordinated voltage control for distribution systems," IEEE PowerTech, Trondheim, pp.1-6, Jun. 2011.

[12] B. Gong and I.A. Hiskens, "Two-stage model predictive control for voltage collapse prevention," Proceedings of the 40th North American Power Symposium, Calgary, Canada, September 2008.

[13] M.M. Abidi and D.P. Milanics, "Reactive capability limitation of synchronous machines," IEEE Transactions on Power Systems, vol. 9, no. 1, pp. 29 - 40, Feb. 1994.

[14] T. Van Cutsem and C. Vournas, Voltage Stability of Electric Power Systems. Boston, MA: Kluwer (now Springer), 1998.

[15] United Kingdom Generic Distribution Network (UKGDS). [Online]. Available: http://sedg.ac.uk.

[16] C. Vournas, E. Potamianakis, C. Moors and T. Van Cutsem, "An educational simulation tool for power system control and stability," IEEE Transactions on Power Systems, vol. 19, no. 1, pp. 48-55, Feb. 2004.

[17] K. Holmstrom, A. Goran and M. Edvall, "User's Guide for TOMLAB 7," available at http://tomopt.com/tomlab, May 2010.

[18] J. D. Hurley, L. N. Bize, and C. R. Mummert, "The adverse effects of excitation system var and power factor controllers," IEEE Transactions on Energy Conversion, vol. 14, no. 4, pp. 16361645, Dec. 1999.

[19] G. Tsourakis, B.M. Nomikos and C. Vournas, "Effect of wind parks with doubly fed asynchronous generators on small-signal stability," Electrical Power System Research, vol. 79, no. 1, pp. 190-200, Jan. 2009.

[20] Power meter specifications: 931A Power System Analyser, Arbiter Systems, Inc. available: http://www.arbiter.com/catalog/product/model-931apower-system-analyzer.php

Gustavo Valverde (S'08-M'12) obtained the B.Sc. degree in Electrical Engineering from the University of Costa Rica in 2005 and the M.Sc. and Ph.D. in Electrical Power Systems from the University of Manchester, UK, in 2008 and 2012, respectively. Currently he is a postdoctoral researcher at the University of Liège, Belgium. His research interests include monitoring of power systems, optimization methods, power system modeling, voltage stability and voltage control of distribution networks.

Thierry Van Cutsem (F'05) graduated in Electrical-Mechanical Engineering from the University of Liège (Belgium), where he obtained the Ph.D. degree and he is now adjunct professor. Since 1980, he has been with the Fund for Scientific Research (FNRS), of which he is now a Research Director. His research interests are in power system dynamics, stability, security, simulation and optimization, in particular voltage stability and security. He is currently chair of the IEEE Power System Dynamic Performance Committee. 